\title{
Integrating Fusion levels for Biometric Authentication System
}

\author{
Darshan R. Kale ${ }^{1}$, Sujata Kulkarni ${ }^{2}$ \\ ${ }^{I}$ ME Scholar, Department of Electronics and Telecommunication Engineering, Mumbai, India \\ ${ }^{2}$ Associate Professor, Department of Electronics and Telecommunication Engineering, Mumbai, India
}

\begin{abstract}
Recently a lot of works are presented in the literature for the multimodal biometric authentication. And such biometric systems have been widely accepted with increasing accuracy rates and population coverage, reducing the vulnerability to spoofing. This paper descripts about the proposed multimodal biometric system that combines the feature extraction level and the score level fusion of iris and face unimodal biometric systems in order to take advantage of both fusion techniques. The experimental results shows the performance of the multimodal and multilevel fusion techniques which are analysed using TRR and TAR to study the recognition behaviour of the approach system. From the ROC Curve plotted, the performance of the proposed system is better compared to the individual fusion techniques.
\end{abstract}

Keywords: Face, iris, identification, unimodal biometric system, multimodal biometric system, feature extraction level, score level.

\section{Introduction}

Finding out the identity of a person has become vital in recent past. A valid/true individual must be able to login to his bank account, ATM, office, computer or any of his own personal accounts. The traditional methods for authentication are password, PIN and token ID or key based. But these methods are insecure as a password can be forgotten or hacked and ID card may be lost or stolen. Biometric system provides sufficient security for the various above stated applications. The word 'Biometrics' refers to the application of statistical analysis of physiological or behavioural characteristics of a person to authenticate his/her identity. In the developing age of nations, the concerns about security and rapid advancement in Networking has been increasing, the demand for a reliable user authentication technique has increased [1].

At present, biometric systems include face characteristics, retina images, finger prints, hand geometry, palm prints, signature, voice etc. Biometrics cannot be forgotten, borrowed, stolen and also forging is impractical. Biometric systems deployed in real world applications are unimodal which rely on the verification of single source of information for authentication. But these systems endure from some of the serious problems like noisy data, intra-class variations, inter-class similarities, non-universality and spoofing. This leads to noticeably high FAR and FRR, limited discrimination capability, upper bound in performance and lack of permanence. To evaluate a biometric system's accuracy, the most commonly adopted metrics are FRR and FAR. FRR is the percentage of authorized individuals rejected by the system and FAR is the percentage of unauthorized persons accepted by the system. The point where FRR and FAR have the same value is called ERR [2]. The limitations of unimodal biometric systems motivated the inclusion of multiple sources of information for establishing identity. A biometric system that fuses the information from multiple traits is known as multimodal biometric system. These systems are more reliable due to the presence of multiple, independent biometrics [2]. Remarkable improvement of the biometric system accuracy can also be further achieved by integrating the fusion level techniques taking the advantage of both the fusion techniques. The proposed biometric system will achieve more accuracy and efficiency than the individual fusion technique.

\section{Literature Survey}

The idea of developing an Integrated Fusion levels for Biometric Authentication System is inspired from the various standard technical papers mentioned below.These papers along with certain research work over the internet has enlighten the various methods and techniques which can be used towards the proposed approach presented in this paper.

\section{A. Related works}

The main idea of combining the fusion levels came from the David et. al [3] combined Feature Extraction Level and Score Level Fusion in which fingerprint and iris are used as traits where advantages of both the fusion techniques are taken. The fusion can be achieved in two different ways. These combined fusion technique performance was better achieved than that of individual fusion techniques. The first is the fusion prior to matching and the second is the fusion after matching. The prior works of research in multimodal biometric systems are reviewed. Fusion at the match score, rank, feature and decision levels has been extensively studied in the literature. D Vaidhya et. al [4] used two modalities: palmprint and palm vein where feature level fusion is used based on the Entropy technique. The system performance is $99 \%$ with FAR $0.02929 \%$, FRR of $1.00 \%$ and accuracy of $99 \%$. Rattani et. al [5] fused face and fingerprints where feature level fusion is performed to enhance the performance of the face and fingerprint modalities alone by $5.05 \%$ and $0.82 \%$ respectively. The feature level fusion outperforms the score level fusion by $0.67 \%$. The system performance i.e. FRR of $1.98 \%$, FAR of 3.18 $\%$ and the system accuracy is $98 \%$. Bahgat et. al [6] proposed a multimodal biometric system by fusing palm vein and face biometric, where feature level fusion is performed. The overall accuracy of the system is more than $95 \%$, FAR of $0.5 \%$, FRR of $1.0 \%$ and accuracy of $98.3 \%$. Feifei et. al [7] fused the fingerprint and finger vein where score level fusion is performed. 
The overall accuracy of the system before fusion fingerprint accuracy is $95.3 \%$, finger vein is $93.72 \%$ and after fusion the accuracy of the system is $98.74 \%$ with FAR of $1.2 \%$, FRR of $0.75 \%$. Nazmeen et. al [8] fused face and ear images in which images are passed to a quality module in order to reduce false rejection rate. Improvement in recognition result is obtained when ear biometric is fused with face biometric. The fusion is done at decision level, achieving a recognition rate of $96 \%$ showing an improvement in accuracy with FAR of $0 \%$ and FRR of 4\%. Nageshkumar et. al [9] fused the palm print and face image in which score level fusion were used and the results are found to be very encouraging and promoting for the research in the field. The overall accuracy of the system is more than 97\%, FAR 2.4\%, FRR 0.8\%. Krzyszof et. al [10] combined the face and speech in which decision level fusion is performed. The system performance is FAR of $3.0 \%$, FRR of $1.1 \%$ and the accuracy of $87 \%$. Mohamad et. al [11] performed multimodal biometrics by fusing the fingerprint and iris in which decision level fusion is used. A fuzzy logic method is used for fusion which is given better performance and accuracy of $98 \%$ with FAR of $2 \%$, FRR of $2 \%$ and accuracy of $98 \%$. Jagadeesan et. al [12] combined the fusion of fingerprint and iris in which feature level fusion is used and the images are pre-processed. The system performance is $91 \%$ with FAR of $10 \%$ and FRR of 5.3\%. Saad Abuguba et. al [13] combined the fusion of iris and face in which feature level fusion is used and cryptographic key is generated. The system performance is FRR of $12.56 \%$ with 256th cryptographic key bit normalization range.

\section{B. Summary}

In the above content, various image fusion techniques are studied and their performances are evaluated on three criteria: Score, Decision and feature fusion. For fusing multiple images feature fusion technique [5, 6, 12, 13] are better choice when compared to score $[7,9]$ and decision $[8,10,11]$ techniques. For proposed work, the feature fusion technique provides a better solution for fusing multiple images and feature sets contains richer information. And also the score level fusion gives the best recognition accuracy results As a result, the combination of feature fusion technique and Score level fusion provides more accuracy and security for fusing multiple images in multimodal biometrics where advantage of both the techniques can be taken [3].

\section{Methodology}

This Paper proposes a similar type of integrated biometric system i.e. face and iris will give higher performance achievement by integrating two fusion techniques i.e. Feature level technique and Score level technique in order to take advantage of both the fusion techniques. The proposed system explains the comparison of feature level \& score level fusion. Such multimodal biometric system will overcome the limitations of Unimodal biometric system and will give better recognition accuracy as compared to the individual traits.

\section{Tools to be used}

The very reason why Iris is selected as the biometric trait is due to its accuracy and easy collectability over networks. But the Face has less performance accuracy as compared to Fingerprint, then-to it has been used by improving its accuracy performance by various algorithms and techniques. To test the proposed system, Experiments will be performed using iris images provided by CASIA Iris Database Version 4.0 collected by the Chinese Academy of Sciences' Institute of Automation which consist of 54601 iris images [15] and face images provided by CASIA-FaceV5 Database of Faces collected by the Chinese Academy of Sciences' Institute of Automation [14]. The proposed Methodology will be carried on MATLAB 2013a software (or any version of MATLAB higher than 2012). The use of Version higher than 2012 is because of the toolbox named Computer-Vision Toolbox is used, which plays an important role in such proposed Biometric Authentication Systems.

\section{Design Methodology}

Figure 1. Shows the flowchart of the proposed project. The input images of face and iris are taken from the databases. These images are pre-processed. Pre-processing of face images will be done as Cropping, resizing, normalization and filtering, whereas median filtering for removal of specular reflections, Iris segmentation, etc. will be for Iris Images.

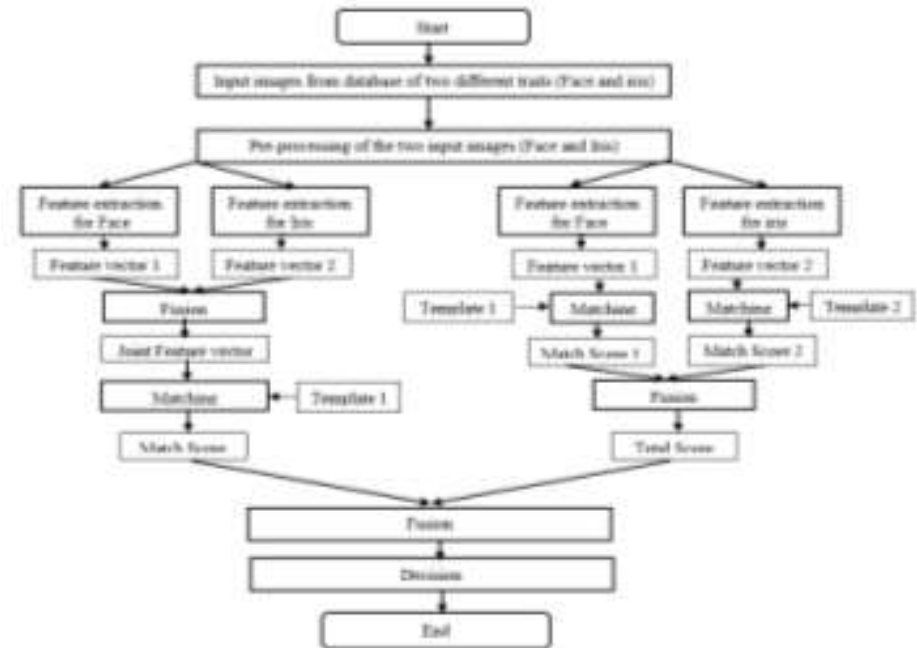

Fig. 1 Block Diagram of Proposed Fusion Biometric System 
Then feature of the face will be extracted with the help of standard and wavelet domain algorithm. After both the feature extraction of images, they are fused at two different fusion level techniques i.e. feature level and Score level fusion. The output of both the fusion technique is then fused i.e. total scores will be calculated through both the techniques. After implementation, the performance can be evaluated and analysed by the parameters like FAR and FRR (or TAR and TRR) with ROC curve. FAR (false rate of acceptance) and FRR (false rate of rejection) are illustrated in [16], [17]. ROC (receiver operating characteristic) [18] curves are methods designed to summarize the performance obtained by biometric detection systems. An ROC curve is a function which indicates, on the x-axis the accepted rate of the impostor attempts and, on the $y$-axis, the accepted rate of genuine attempts using a decision threshold.

First of all, preprocessing of the input images have been done where the term preprocessing term include image resizing, image cropping, image enhancement etc. Then, as the two fusion techniques are been implemented, so the feature extraction techniques used in this proposed project are different so as to get better accuracy and feasibility with respective individual fusion techniques used. Owing towards the Score Level Fusion, Principal component analysis (PCA) for face recognition is based on the information theory approach: it extracts relevant information and encodes it as efficiently as possible. PCA approach reveals the most effective low dimensional structure of facial patterns, removes information that is not useful and specifically decomposes the structure of face into uncorrelated components named Eigen faces [19]. Each image of face may be stored in a 1D array which is the representation of the weighted sum (feature vector) of the Eigen faces. The same is repeated for iris images too. Whereas for Feature level Fusion, The feature extraction technique used for face is by performing LBP operator on images and then applying Gabor filtering on it [20]. For iris images, features are extracted through Circular Hough Transform where circle boundary of iris and pupil are detected through canny edge detector [13], [21] which is a specialization of Hough Transform. And hence the feature vectors are calculated, as concatenation of the vectors has been done which is described below.

\section{E. Feature Level Fusion Scheme}

This first type of fusion (figure 2) is obtained by concatenating the features vectors derived from face and iris images. Compared to individual vectors, the obtained concatenated vector of features has an increased power of discrimination. There are many types of algorithms used for extraction process. For face, LBP operator: Local binary patterns were introduced by Ojala et al. [20], [25] as a fine scale texture descriptor. In its simplest form, an LBP description of a pixel is created by thresholding the values of the $3 \times 3$ neighbourhood of the pixel against the central pixel and interpreting the result as a binary number. Then Gabor filtering has been applied to get unit magnitude of a feature vector with a down-sampling filter bank. For iris, the iris and pupil boundary edge map has been detected by canny edge detection technique $[13,21]$ and the Hough circle of an eye has been extracted through it. The mean, Entropy and Variance of the extracted feature has been calculated and concatenated with respect to users. And hence the final vector has been formed by concatenation. In order to create this concatenated vector we used the process of fusing biometric data. There, concatenation of the iris " $\mathrm{I}(\mathrm{t})$ " feature vector and face " $\mathrm{F}(\mathrm{t})$ " feature vector is obtained i.e. a new vector "V(t)" (1), using the formula:

$V(t)=F(t)+I(t)$

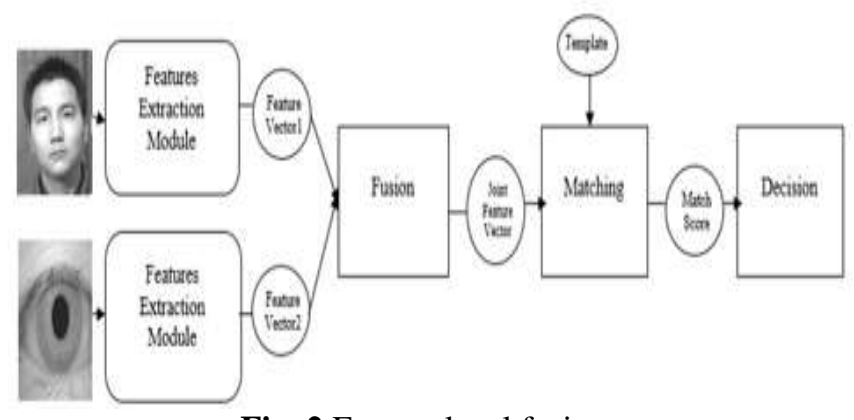

Fig. 2 Feature level fusion

After that for person authentication, measurement of the Euclidean distance between the current vector "V(t)", and all the vectors "V(t)" from our registered users, existing in the database is done. In order to distinguish between an enrolled used and an impostor used in the decision process, a certain threshold ( $t)$ is used. 


\section{F. Score Level Fusion Scheme}

This second type of fusion (figure 4) is often used, as scores derived by each unimodal biometric system, usually contain enough biometric information to make a difference between a registered user and an intruder. For each biometric trait a match score is provided and at the end of the fusing process the scores are combined. Finally the acceptance or the rejection decision is taken. The way information is combined makes this kind of biometric systems both feasible and practical [11].

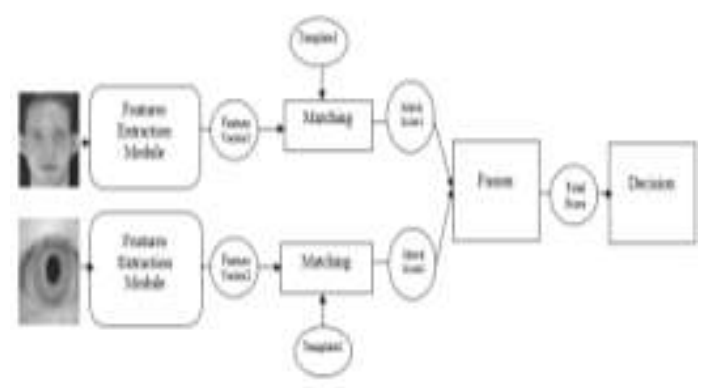

Fig. 3 Score Level Fusion

Here Eigen Face Based Approach have been used. Principal component analysis (PCA) is used to extract Eigen faces [26]. Initially Eigen vectors are computed using covariance matrix derived from set of training images. Probe image is then projected in to the face space and the distance between mean Eigen face and probe image is computed using spatial differential operators such as Euclidian distance. For the recognition of face biometrics, face images are normally projected into the feature space which best encodes the variation of the image. This feature space is also known as the Eigen-face, which is the eigenvector of the set of faces.

Suppose we have Eq. below:

$X_{i}=\left[S_{i} \ldots \ldots . S_{n}\right]^{T}$

where $\left[S_{i} \ldots \ldots S_{m}\right]^{T}$ represents the input signal of the face images and $i=1 \ldots \ldots$. During empirical mean detection and calculation phase, the face images are being mean centred by subtracting the mean image from each image vector. The mean, $v$, will represent the mean image as Eq. below:

$v=\frac{1}{n} \sum_{i=1}^{n} X_{i}$

where the mean centred image is $X_{i}-v$. Next, the eigenvectors and eigenvalues calculation process being executed. Eigenvectors of the covariance matrix, $\mathrm{Y}(m \times n)$ give the Eigen faces, where $\mathrm{Y}=X X^{T}$ are generated, and these eigenvectors are sorted from high to low following the eigenvalues calculated from the covariance matrix. The highest eigenvalues give the largest variance in the image. The training sets of face images were acquired and the Eigen faces were calculated using Principal Component Analysis (PCA) projections. A 2-D facial image was represented as a 1-D vector. Each of the Eigen faces can be viewed as a feature and is expressed by Eigen face coefficients (weight).

Then the matching of face and iris images was done by retrieving the enrolled image from the dataset and calculated using the Weighted Euclidean Distance, Eq. below with the image of the tester subjects.

$W E D=\sqrt{\sum_{i=1}^{n} W_{i}\left(A_{i}-B_{i}\right)^{2}}$

where A and B represents the enrolled and testing biometric images. The above procedure is repeated for the iris images too. The fusing score can be computed as in Eq.

$S_{\text {fussdissors }}=\sum_{j=1}^{w}\left(W_{1, j} S_{i S c o r s}+W_{2, j} S_{f S c o r s}\right)$ 
Where $S_{i S \text { cors }}$ and $S_{f S c o r e}$ are the normalized scores of the biometric matchers face and iris respectively. Depending on the weight of the face and iris biometrics, if both of them were equal, Eq.(5) can be derived or simplified into Eq.

$S_{\text {fussdiscore }}=\sum_{j=1}^{y}\left(S_{\text {iScore }}+S_{f S c o r e}\right)$

Through the steps described in the following section, the unified fusing score $S_{\text {fwsedscors }}$, was evaluated based on the pre-specified threshold value, ${ }^{\tau}$. The ${ }^{\tau}$ value is defined based on the average value obtained for the overall results. We declared the user to be genuine when $S_{\text {fussdiscors }} \leq \tau$, otherwise the user was an impostor [27].

\section{Proposed Final Combined System}

Here we propose a fusion technique which combines the first and the second type of fusion Scores which are described in section $\mathrm{E}$ and $\mathrm{F}$.

As described in equation (6), the scores are fused and the decision is taken. Finally the acceptance or the rejection decision is taken. Here AND rule is used for the decision. The way information is combined makes this kind of biometric systems both feasible and practical. Since traits are not from the same person, aim is to design the prototype of multimodal system. Using the proposed system we take advantage of both features level fusion techniques and score level fusion techniques, and hence we achieve a stronger biometric system which is more accurate and efficient.

\section{Experimental Results}

The following are the results obtained which includes few GUI of the proposed system with time complexity of the each step performed in the system.

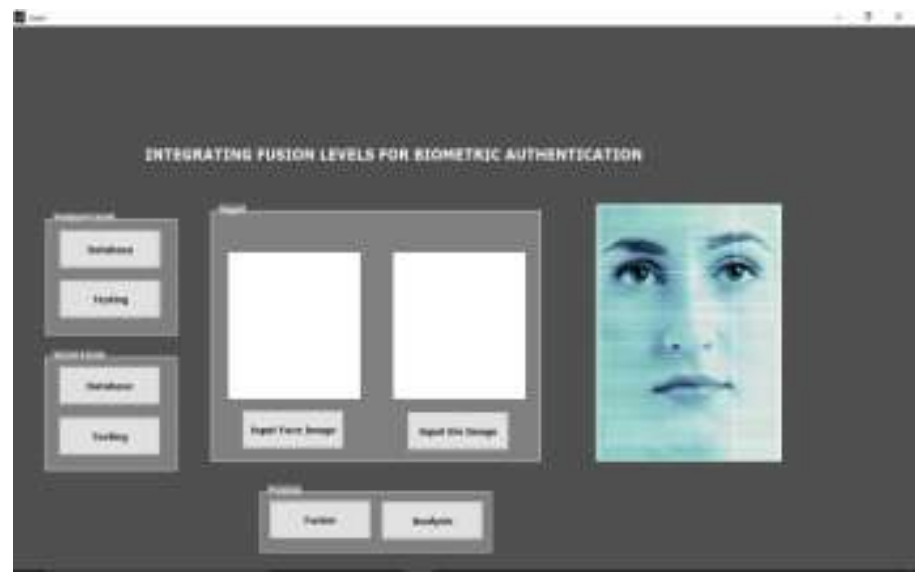

Fig. 4 GUI of proposed system

The Figures 5-9 illustrates the working of the proposed system and their time complexity respectively. Firstly the database creation in the feature level fusion and secondly the matching of the input data with the data created in the database.

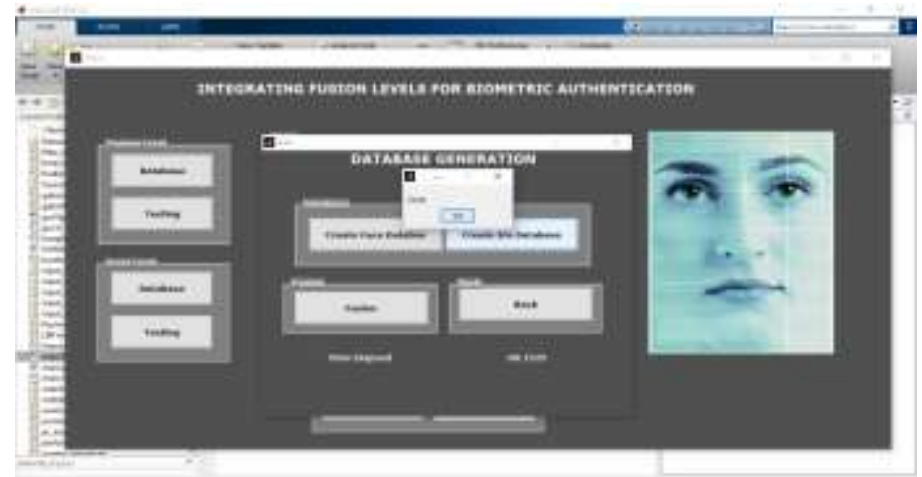

Fig. 5 Feature level database creation of face with time complexity 

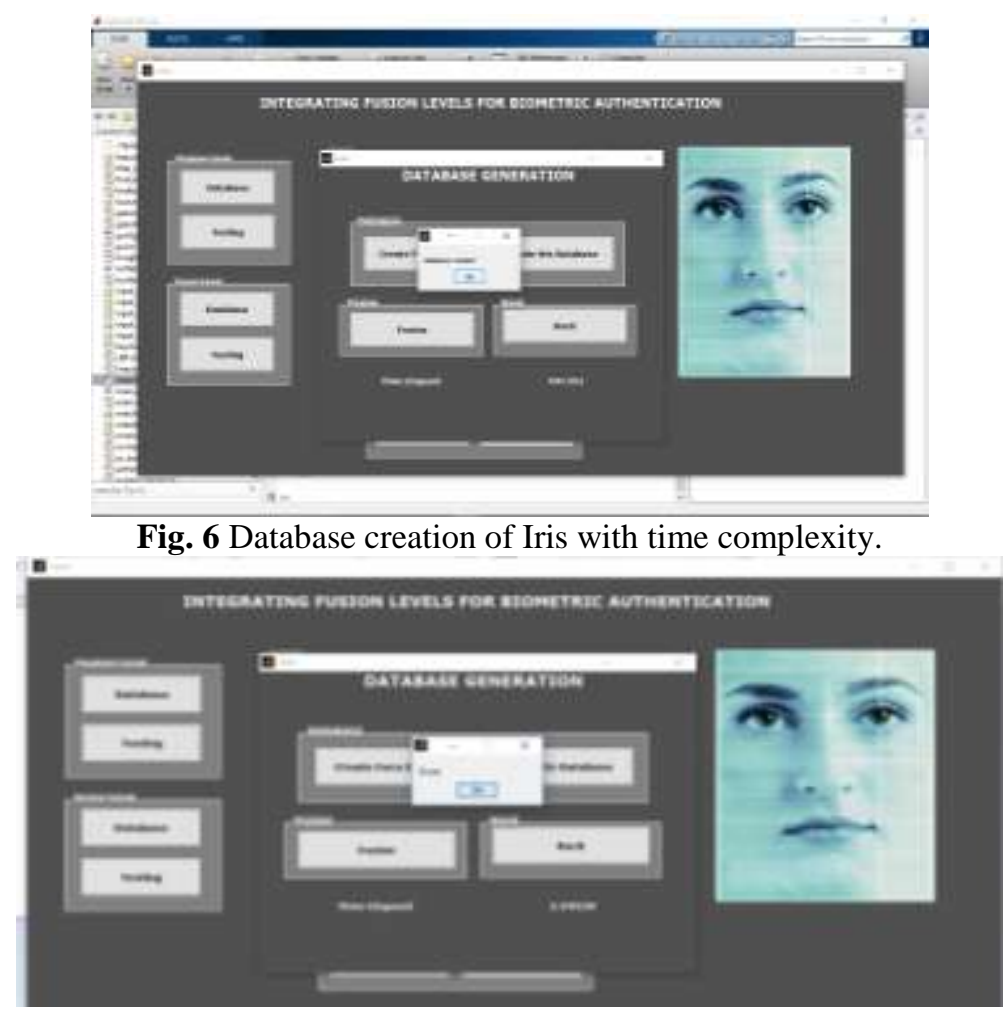

Fig. 7 Fusion of face and iris with time complexity

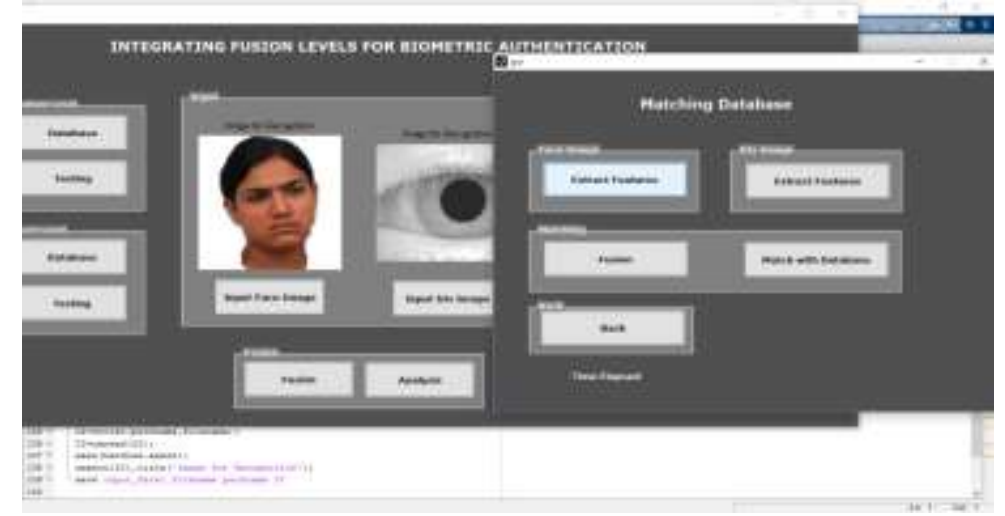

Fig. 8 Verification phase (testing)

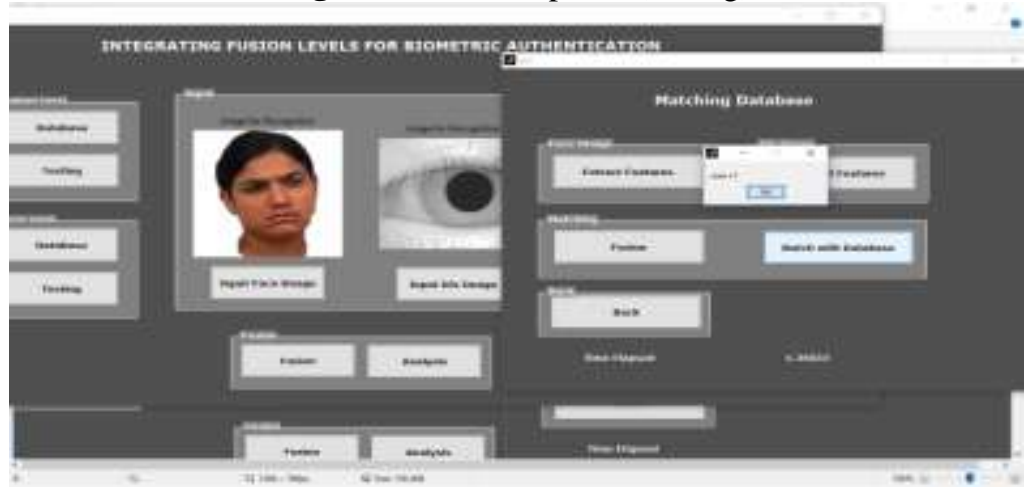

Fig. 9 Verification of user as 1 from database created earlier with time complexity

Hence, the above GUI figures show the working of proposed methodology with its time complexity, where the person is verified as a registered user in the database. And so the biometric authentication system gains the stronger security in terms of integrating fusion levels which is the proposed methodology. The result showing ROC curves are shown below which describes the total objective of the proposed system. 


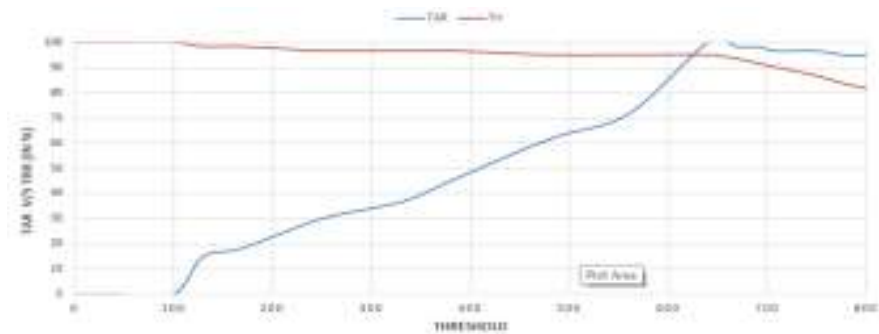

Fig. 10 Recognition Accuracy using Feature level fusion=94\%

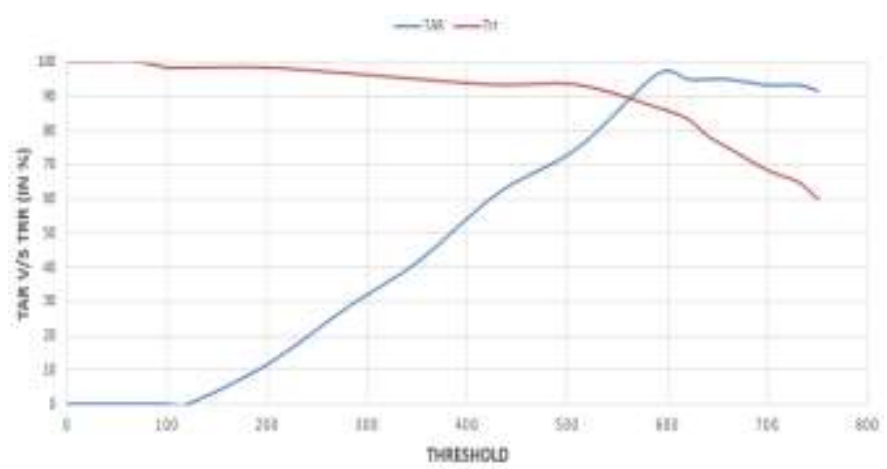

Fig. 11 Recognition accuracy using Score level fusion= $91.35 \%$

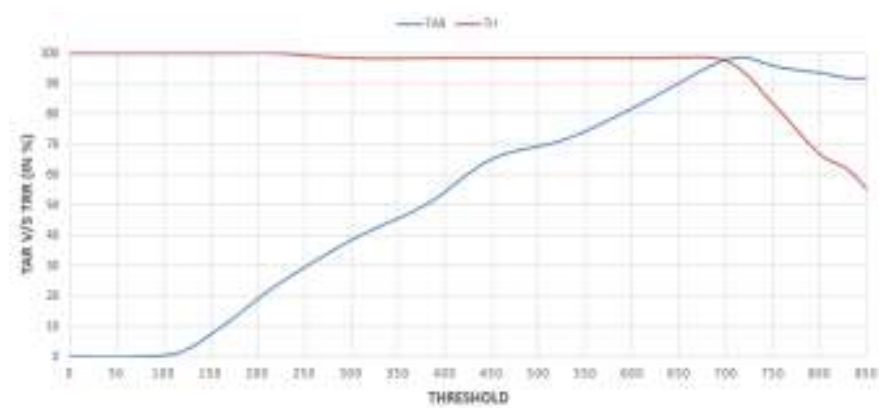

Fig. 12 Recognition accuracy of final fusion $=97 \%$ AOC CURVE

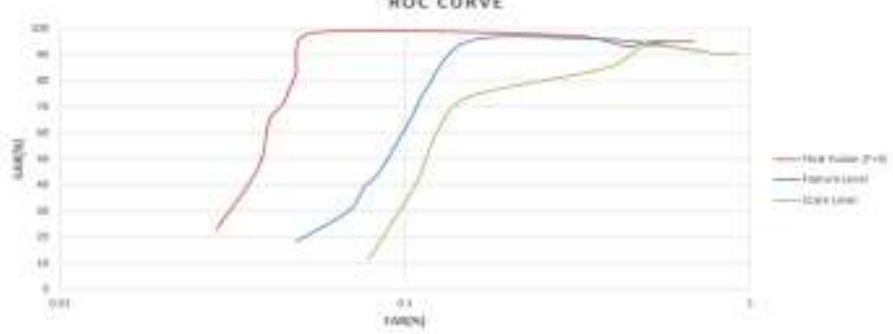

Fig. 13 ROC Curve - GAR (\%) V/s FAR (\%)

Hence the above presented result is obtained for multimodal biometric fusion at first level (Feature level) with 94\%, second level (Score level) with $91.35 \%$ and the proposed method with $97 \%$ recognition accuracy. These results proves that the proposed biometric system overcomes the performance given by the individual fusion techniques respectively with a significantly increasing recognition accuracy. Here the True Rejection Rate i.e. TRR of the proposed method has been achieved better as compared to the two individual fusion techniques used.

The time complexity as shown in the above various results have been tabulated below with respect to the two different fusion techniques i.e. Feature level and Score level:

Table 1 Time complexity of the proposed system

\begin{tabular}{|l|l|l|}
\hline Time taken (s) & Feature level fusion & Score level fusion \\
\hline & Training & \\
\hline Feature extraction for face & 60.15 & 0.57 \\
\hline Feature extraction for iris & 4106.75 & 0.46 \\
\hline Fusion & 3.95 & NA \\
\hline & Testing & \\
\hline Feature extraction for face & 5.30 & 0.051 \\
\hline Feature extraction for iris & 43.35 & 0.044 \\
\hline
\end{tabular}




\begin{tabular}{|l|l|l|}
\hline Fusion & 0.38 & 0.005 \\
\hline Matching & 6.73 & Face-0.07 \\
\cline { 3 - 3 } & & Iris-0.07 \\
\hline
\end{tabular}

\section{Conclusion And Future Scope}

The Recognition accuracy of the proposed system is $97 \%$ which is more than the individual fusion techniques i.e. $94 \%$ of Feature level and $91.35 \%$ of Score level, which shows that the proposed fusion technique has better performance than the individual fusion techniques. As the scope of using multimodal biometric system has become widen due to its performance which overcomes the performance of the unimodal system. The proposed method combines the two fusion techniques and the experimental results obtained shows that the proposed system gives stronger security than the individual fusion techniques. The system achieves a stronger security stopping the intruder from any attack.

Here, the merits of final integrated fusion technique has been highlighted. The future scope can be more evolved by using different traits and by different fusion techniques.

\section{Acknowledgement}

The Authors highly appreciate the Contribution of CASIA (Chinese Academy of Sciences' Institute of Automation) as the portion of the research in this paper uses the CASIA-IrisV4 and CASIA-FaceV5 as well.

\section{References}

[1] K. Jain, A. Ross, S. Prabhakar, "An introduction to biometric recognition", IEEE Transactions on Circuits and Systems for Video Technology, vol. 14, pp. 4-20, 2004

[2] Sudhamani M J, M K Venkatesha, K R Radhika, "Revisiting Feature level and Score level Fusion Techniques in Multimodal Biometrics System", International Conference on Multimedia Computing and Systems (ICMCS), Tangier, May 2012.

[3] David Marius Daniel, Cîslariu Mihaela, Terebes Romulus, "Combining Feature Extraction Level and Score Level Fusion in a Multimodal Biometric System"11th ISETC-International Symposium on Electronics and Telecommunications, pp. 1-4, 2014.

[4] D Vaidhya, S pawar, "Feature level fusion of palmprint and palm vein for personal authentication based on Entrophy technique", International Journal on Electronics and communication Technology, Vol.5, Issue spl-1, 2014.

[5] A.Rattani, D.R.Kishu, M.Bicego," Feature level fusion of face and fingerprint Biometrics", Biometrics: Theory, applications and systems, First IEEE International conference, 2007

[6] S.F.Bahgat, S.Ghoniemy, M.Alotabi," Proposed Multimodal palm-veins- face biometric Authentication", International journal of advanced computer science and applications, vol-4, No.6, 2013.

[7] Feifei cui, Gongping yang, "Score level fusion of fingerprint and finger vein Recognition", Journal of Computer Information's systems: 16, pp. 5723-5731, 2011.

[8] Nazmeen bibi boodoo, R.K.Subramanian, "Robust multi-biometric recognition using face and ear images", IJCSIS-International journal of computer science and information security, Vol.6, No.2, 2009.

[9] Nageshkumar, Mahesh.PK, Shanmuka swami M.N, "A Efficient Multimodal biometric fusion using palmprint and a face image", International Journal of computer science, Vol.2, Issue 3, 2009.

[10] Krzyszof, Jonas Richard, Plamen Prodanov, Andrzejn Drygajlo, "Reliability- Based decision fusion in multimodal biometric verification systems", EURASIP-European Journal on advances in signal processing, Article ID 86572, 9 Pages, 2007.

[11] Mohamad Abdolahi, Majid Mohamadi, Mehdi Jafari, "Multimodal biometric system fusion using fingerprint and iris with fuzzy logic", International Journal of soft computing and engineering, vol.2, Issue-6, 2013.

[12] A.Jagadessan, Dr.K.Duraisamy, "Secured Cryptographic Key Generation from multimodal biometrics: Feature level fusion of fingerprint and Iris", International Journal of computer science and information security, Vol.7, No.2, 2010.

[13] Saad Abuguba, Milan M. Milosavljević, Nemanja Maček, "An Efficient Approach to Generating Cryptographic Keys from Face and Iris Biometrics Fused at the Feature Level", IJCSNS International Journal of Computer Science and Network Security, vol.15 No.6, June 2015

[14] Chinese academy of sciences, CASIA Face image database Version 5.0, http://biometrics.idealtest.org

[15] Chinese academy of sciences, CASIA Iris image database Version 4.0, http://biometrics.idealtest.org

[16] Sahoo, Soyuj Kumar, Mahadeva Prasanna, SR, Choubisa, Tarun, "Multimodal Biometric Person Authentication: A Review", IETE Technical, Page 54, 2012.

[17] Ravi Das, "An introduction to biometrics", Keesing Journal of Documents \& Identity, Issue 17, Page 5, 2006

[18] A. J. Mansfield, J. L. Wayman, "Best Practices in Testing and Reporting Performance of Biometric Devices", Centre for Mathematics and Scientific Computing National Physical Laboratory Queens Road Teddington, Page 7, 2002.

[19] M. A. Turk and A. P. Pentland, "Face recognition using eigenfaces", In Proc. IEEE Computer Society Conf. Computer Vision and Pattern Recognition, Maui, Hawaii, pp.586-591, 1991.

[20] D Sharma and Dr. A. Kumar, "An Empirical Analysis Over the Four Different Feature-Based Face and Iris Biometric Recognition Techniques”, (IJACSA) International Journal of Advanced Computer Science and Applications, vol. 3, No. 10, 2012.

[21] G. Amoli, N. Thapliyal and N Sethi, "Iris Preprocessing", (IJARCSSE) International Journal of Advanced Research in Computer Science and Software Engineering, vol. 2,issue 6,2012.

[22] P. D. Garje and S. S. Agrawal, "Multibiometric Identification System Based On Score Level Fusion" IOSR Journal of Electronics and Communication Engineering (IOSRJECE), Volume 2, Pages 07-11, Issue 6, 2012

[23] G.L. Marcialis, F. Roli, "Score-level fusion of fingerprint and face matchers for personal verification under stress conditions", the $14^{\text {th }}$ International Conference on Image Analysis and Processing, , Pages 259-264, 2007.

[24] Ross and A.K. Jain, "Information fusion in biometrics-Pattern Recognition Letters", Pages 2115-2125, 2003.

[25] T. Ojala, M. Pietikainen and D. Harwood, "A comparative study of texture measures with classification based on feature distributions",Pattern Recognition, vol.29, no.1,pp. 51-59, 1996.

[26] Tanaya MandaI and Q. M. Jonathan Wu, "Face Recognition using Curvelet Based PCA," 19th International Conference on Pattern Recognition,8-11 December 2009, vol. 89, pp. 2345-2353.

[27] H. M. Sim,H. Asmuni, R. Hassan, R. M. Osman, "Multimodal biometrics: Weighted score level fusion based on non-ideal iris and face images",Elseiver-Expert systems with applications 41(2014),pp. 5390-5404. 